CARDIOVASCULAR MEDICINE

\title{
Plasma von Willebrand factor, soluble thrombomodulin, and fibrin D-dimer concentrations in acute onset non- rheumatic atrial fibrillation
}

\author{
F Marín, V Roldán, V E Climent, A Ibáñez, A García, P Marco, F Sogorb, G Y H Lip
}

Heart 2004;90:1162-1166. doi: 10.1136/hrt.2003.024521

See end of article for authors' affiliations

Correspondence to

Dr F Marín, Department of

Cardiology, General

Hospital of Alicante, Pintor

Baeza $\mathrm{s} / \mathrm{n}$, Alicante

03002, Spain;

fcomarino@hotmail.com

Accepted

18 December 2003
Objective: To investigate whether new onset acute atrial fibrillation (AF) of $<48$ hours' duration creates a prothrombotic state in the absence of anticoagulation and to assess the evolution in research indices after spontaneous or pharmacological cardioversion.

Methods: 24 patients were recruited with first onset acute non-rheumatic AF, in whom sinus rhythm was restored within 48 hours of arrhythmia onset, without anticoagulant treatment. Atrial mechanical function was assessed by transmitral inflow. Soluble thrombomodulin and von Willebrand factor concentrations (both as indices of endothelial damage or dysfunction) and fibrin D-dimer concentrations (as an index of thrombogenesis) were measured. Blood samples were drawn and echocardiographic studies were performed at days 1, 3, 7, and 30 after cardioversion. Research indices were compared with those of 24 healthy participants, 24 patients with chronic AF, and 24 patients with ischaemic heart disease in sinus rhythm.

Results: Patients with AF had higher concentrations of soluble thrombomodulin (acute AF 12.1 (4.1) ng/ml; chronic AF $11.8(4.6) \mathrm{ng} / \mathrm{ml}$ ), von Willebrand factor (acute AF 137.2 (36.9) ng/ml; chronic AF 133.1 (25.0) $\mathrm{ng} / \mathrm{ml}$ ), and fibrin D-dimer concentrations (acute AF $2.35(2.68) \mu \mathrm{g} / \mathrm{ml}$; chronic AF $1.12(0.65) \mu \mathrm{g} / \mathrm{ml}$ ) than did healthy controls $(5.9(2.7) \mathrm{ng} / \mathrm{ml}, 86.7(33.2) \mathrm{ng} / \mathrm{ml}$, and $0.39(0.28) \mu \mathrm{g} / \mathrm{ml}$, respectively) and patients with ischaemic heart disease $(7.4(3.7) \mathrm{ng} / \mathrm{ml}, 110.0(29.0) \mathrm{ng} / \mathrm{ml}$, and 0.99 (0.73) $\mu \mathrm{g} / \mathrm{ml}$, respectively) (all $p<0.05$ ). Day 30 concentrations offibrin D-dimer were higher in patients with acute AF than in patients with chronic AF ( $p=0.038$ ) but sTM and von Willebrand factor concentrations were not different (both not significant). There were no significant changes in research indices or echocardiographic parameters after cardioversion (all $p>0.05$ ).

Conclusions: There was evidence among patients with acute onset AF of endothelial damage or dysfunction and increased thrombogenesis, which persisted up to 30 days after cardioversion.
$\mathrm{P}$ atients with chronic atrial fibrillation $(\mathrm{AF})$ are considered to be at high risk of systemic thromboembolism, which is reduced by anticoagulation. In the presence of risk factors, patients with paroxysmal and chronic AF appear to have a similar risk for thromboembolic stroke, regardless of the time spent in AF. ${ }^{1}$ Nevertheless, the time required for thrombus formation in $\mathrm{AF}$ is uncertain, although it is generally accepted that an atrial thrombus is formed $48-72$ hours after the onset of acute arrhythmia. ${ }^{2}$ Thus, current treatment guidelines suggest that an anticoagulant be initiated for patients with $\mathrm{AF}$ for $>2$ days (or if the onset is uncertain or unknown) for a minimum of three weeks before elective cardioversion. ${ }^{3}$ However, limited trial data are available about the need for anticoagulation in patients with AF of $<48$ hours' duration.

There is increasing evidence that AF may confer a prothrombotic or hypercoagulable state, with abnormal haemostasis, fibrinolysis, platelets, and endothelial function. ${ }^{4-9}$ However, previous studies relate mainly to the setting of chronic $\mathrm{AF}$ in patients with permanent $\mathrm{AF}^{5-7}$ and in patients with paroxysmal (recurrent) AF. ${ }^{8}{ }^{9}$ Few studies have prospectively assessed the presence of a prothrombotic state in the setting of acute AF, especially in the absence of anticoagulation treatment.

The restoration of sinus rhythm is associated with the recovery of atrial contraction, although this may not necessarily occur immediately after conversion to sinus rhythm. ${ }^{10}$ Cardioversion of AF can also be associated with a transient mechanical dysfunction of the left atrium called "atrial stunning". ${ }^{11}$ Both factors increase atrial stasis (evident from the increased spontaneous echo contrast) and abnormal haemostasis, which therefore fulfil components of Virchow's triad, leading to the development of atrial thrombus and thromboembolism after cardioversion. ${ }^{4}{ }^{12} 13$

The aim of the present study was to investigate whether new onset acute AF of $<48$ hours' duration creates a prothrombotic state (as reflected by measurement of soluble thrombomodulin (sTM) and von Willebrand factor (vWf) concentrations (both as indices of endothelial damage or dysfunction) and of fibrin D-dimer concentrations (as an index of thrombogenesis $))^{14}$ in the absence of anticoagulation and to assess the evolution in research indices after spontaneous or pharmacological cardioversion. We therefore prospectively studied 24 patients with new onset acute AF. Their blood was sampled and Doppler echocardiography performed at days 1, 3, 7, and 30 after reversion to sinus rhythm.

\section{PATIENTS AND METHODS}

For this study, we applied a (very) strict definition of acute $\mathrm{AF}$ and prospectively recruited only consecutive patients with first onset acute $\mathrm{AF}^{15}$ who were certain of arrhythmia onset

Abbreviations: AF, atrial fibrillation; ELISA, enzyme linked immunosorbent assay; sTM, soluble thrombomodulin; vWf, von Willebrand factor 
within 48 hours of presentation. These patients had to be free of AF recurrence for 30 days after inclusion in the study. We also excluded patients older than 70 years, as well as patients with rheumatic valvar heart disease; previous venous thrombosis or history of systemic thromboembolism; recent $(<3$ months $)$ surgery, acute infection, or inflammatory disease; malignancy; recent ( $<3$ months) myocardial infarction, acute coronary syndrome; or stroke; diabetes, renal or liver impairment, or obesity; excess alcohol intake; or current treatment with oral anticoagulant or hormone replacement therapy. Patients with previously documented episodes of AF were excluded.

Over the study period, 41 consecutive patients with new onset acute AF fulfilled the inclusion criteria. Of these, 32 patients were successfully restored to sinus rhythm by spontaneous or pharmacological cardioversion within 48 hours of AF onset. The time to cardioversion was taken as the time from initial presentation of symptoms to the time of successful cardioversion. During follow up, eight patients had a relapse of AF and were excluded from the analysis, as their AF could now be defined as paroxysmal or persistent, requiring further cardioversion. ${ }^{15}$ Thus, the study group of patients with acute AF (strictly defined, for the purposes of this study) consisted of 24 patients. Blood was sampled and Doppler echocardiographic studies were performed at days 1, 3, 7, and 30 after cardioversion. None of the patients was receiving anticoagulation. Four patients were being treated with aspirin before the onset of AF.

Blood sample results from patients with AF at 30 days after cardioversion (in sinus rhythm) were compared with results from 24 age and sex matched healthy participants who were in sinus rhythm. This healthy control group was drawn from among healthy hospital staff and from patients attending hospital for hernia repair or minor operations. All were free of diabetes and had no signs or symptoms of cardiovascular, cerebrovascular, neoplastic, or connective tissue disease. We also studied two other control groups for the cross sectional comparison. Firstly, we recruited 24 patients in chronic AF from our clinic (defined as patients for whom cardioversion had been deemed inappropriate, with AF confirmed on multiple ECGs) who were not given anticoagulation, although 10 were taking aspirin at the time of blood sampling. Secondly, we studied 24 "vascular disease controls" who were patients with previous myocardial infarction in sinus rhythm and with no previous documented AF. All of this last group were taking aspirin. Patients and controls were fully informed of the aim of this study. All study patients and participants gave their informed consent to enter the study.

\section{Blood samples and laboratory assays}

Venepuncture was performed in the morning after patients had been fasting for $>12$ hours and had rested for at least 20 minutes. Blood samples were drawn atraumatically and without stasis into syringes preloaded with trisodium citrate $(0.011 \mathrm{M})$. Platelet poor plasma fractions were obtained by centrifugation at $4^{\circ} \mathrm{C}$ for 20 minutes at $2200 \mathrm{~g}$ (within five minutes after blood collection). Aliquots were stored at $-20^{\circ} \mathrm{C}$ to allow for batch analysis.

We measured sTM and vWf concentrations by enzyme linked immunosorbent assay (ELISA) (Diagnostica Stago, Paris, France). Fibrin D-dimer concentrations were measured with a LIA-DD test on an STA coagulometer (Boehringer Mannheim, Mannheim, Germany). Intra-assay coefficients of variation for all ELISAs were $<5 \%$ and interassay variances were $<10 \%$.

\section{Echocardiographic studies}

Transthoracic M mode, two dimensional, and Doppler echocardiography (Sonos 2500 ultrasonograph, Hewlett Packard, Palo Alto, California, USA) was performed at days $1,3,7$, and 30 after successful cardioversion. Pulsed Doppler transmitral inflow velocities were recorded from the apical four chamber view with the sample volume positioned between the tips of the mitral leaflets. Peak velocities of the early filling ( $\mathrm{E}$ wave) and atrial filling (A wave) were measured. The velocity-time integral of the transmitral inflow was determined. The atrial mechanical contribution to total left ventricular filling was assessed by dividing the area under the A wave by the total area under the diastolic velocity-time curve. Thus, E:A and the A wave velocity-time integral to total velocity-time integral ratio $(\mid \mathrm{A} / / \mathrm{T})$ were calculated at each study time point. All echocardiograms were recorded by the same investigator and the coefficient of variation for our laboratory was $<5 \%$.

\section{Power calculations and statistical analysis}

We hypothesised that STM would be increased by one quarter of a standard deviation among patients with AF compared with controls. To prove this we would have needed to recruit a minimum of 20 patients and 20 controls for a one sided $\mathrm{p}<0.05$ with a power of $80 \%$. Previous studies of hypercoagulability and cardioversion of AF recruited only seven ${ }^{16}$ and 23 patients, ${ }^{17}$ whereas in our series 24 patients remained in sinus rhythm at day 30 , in keeping with our strict definition of acute onset AF.

Continuous variables were tested for normal distribution with the Kolmogorov-Smirnov test. Results are expressed as mean (SD). Differences between patient and control data were analysed by one way analysis of variance with Tukey's post hoc analyses for intergroup comparisons. Sequential data after acute AF were analysed by repeated measures analysis of variance. Correlations were tested by Spearman's rank correlation method. All calculations were done with the SPSS for Windows software (SPSS Inc, Chicago, Illinois, USA). A probability value of $\mathrm{p}<0.05$ was considered significant in all statistical analyses.

\section{RESULTS}

We prospectively studied 24 patients ( 12 men, mean age 64 (3) years, range 59-68 years) with first onset acute nonrheumatic $\mathrm{AF}$ in whom sinus rhythm was restored by spontaneous $(\mathrm{n}=15)$ or pharmacological cardioversion ( $\mathrm{n}=9$; six with amiodarone and three with flecainide) within 48 hours of arrhythmia onset, with no anticoagulant treatment. Four patients had underlying hypertension (> 140/90 mm Hg), four had chronic ischaemic heart disease (defined by standard criteria), one had mild aortic valve disease (aortic valve area $1.5 \mathrm{~cm}^{2}$ ), and one had hypertrophic cardiomyopathy. All the patients with acute AF had a normal left atrial dimension on $\mathrm{M}$ mode echocardiography (mean $38.0 \mathrm{~mm}$, range $34.3-40.8 \mathrm{~mm}$ ) and left ventricular function (mean ejection fraction $71 \%$, range $61-79 \%$ ).

Table 1 lists clinical data for the four study groups. Patients 30 days after acute $\mathrm{AF}$ and those with chronic AF had significantly higher mean STM, vWf, and fibrin D-dimer concentrations than did healthy controls and vascular disease controls in sinus rhythm (all p $<0.05$ ). There were no significant differences in STM and vWf concentrations between the acute AF and chronic AF patient subgroups. Significantly higher D-dimer concentrations were found in patients with acute AF than patients with chronic AF at day $30(\mathrm{p}=0.038)$ but other indices were not significantly different (table 2).

There were no significant changes in echocardiographic parameters and research indices after cardioversion of acute 


\begin{tabular}{lllll}
\hline Table 1 Clinical data of the four study groups & & \\
\hline & Acute AF & Chronic AF & IHD & $\begin{array}{l}\text { Healthy } \\
\text { controls }\end{array}$ \\
\hline Number & 24 & 24 & 24 & 24 \\
Mean (SD) age (years) & $64(3)$ & $64(5)$ & $58(10)$ & $63(6)$ \\
Male sex & 12 & 11 & 14 & 12 \\
Medical history & 4 & 6 & 8 & 0 \\
$\quad$ Hypertension & 0 & 7 & 4 & 0 \\
$\quad$ Diabetes & 4 & 3 & 24 & 0 \\
$\quad$ Myocardial infarction & 4 & 10 & 24 & 0 \\
Drugs & 0 & 8 & 0 & 0 \\
Aspirin & 4 & 2 & 16 & 0 \\
$\quad$ Digoxin & 5 & 3 & 9 & 0 \\
$\quad$ B Blocker & 4 & 7 & 5 & 0 \\
ACE inhibitors & & & \\
CCB & 4 & & \\
\hline ACE, angiotensin converting enzyme; AF, atrial fibrillation; CCB, calcium channel blocker; IHD, ischaemic heart \\
disease.
\end{tabular}

AF (repeated measures analysis of variance, all $p>0.05$ ) (table 3), although there was a non-significant trend towards a reduction in fibrin D-dimer concentrations $(p=0.066)$. There were no significant correlations between sTM, vWf, and fibrin D-dimer concentrations with echocardiographic or Doppler indices (Spearman, p > 0.05, data not shown).

\section{DISCUSSION}

The present study suggests that in the absence of anticoagulation, patients with acute onset AF at day l after cardioversion have endothelial damage or dysfunction (sTM, vWf) and increased thrombogenesis (fibrin D-dimer), which do not appear to be significantly altered after cardioversion, at least up to day 30. Furthermore, new onset acute AF of $<48$ hours' duration does not appear to cause significant left atrial stunning or activation of the prothrombotic state related to the acute episode. For new onset AF, no randomised controlled trials exist to make specific recommendations about anticoagulation treatment but the present study suggests that acute AF may also create a prothrombotic or hypercoagulable state. ${ }^{4}$

Transient atrial dysfunction (referred to as atrial stunning) has been described in patients with AF after the recovery of sinus rhythm. ${ }^{18}$ This phenomenon is considered an important mechanism responsible for thromboembolism after both electrical and pharmacological cardioversion. ${ }^{12} 1519$ Indeed, new or increased atrial spontaneous echo contrast ${ }^{13}$ or new atrial thrombus formation after cardioversion has been related to this phenomenon. ${ }^{20}$ The degree of atrial stunning appears to depend on several factors, such as the duration of the arrhythmia, mode of cardioversion, or the presence of structural heart disease. ${ }^{21-23}$ Nonetheless, significant left atrial stunning does not seem to occur after only a brief duration of $\mathrm{AF}^{12}$ and the present study supports this assumption. The restoration of atrial mechanical function is also related to the prior duration of AF. Patients with AF lasting less than two weeks would have complete restoration of atrial mechanical function within 24 hours. ${ }^{24}$ Indeed, in the present study we did not observe any significant increase in the A wave or in E:A.

Patients after acute AF and those with chronic AF had significantly higher mean sTM, vWf, and fibrin D-dimer concentrations than did healthy controls in sinus rhythm, indicating endothelial damage or dysfunction and abnormal thrombogenesis. Indeed, new onset acute AF and chronic AF appear to have similar abnormalities in prothrombotic indices. ${ }^{192526}$ Importantly, Heppel and colleagues ${ }^{27}$ reported an association between prothrombotic indices and the presence of left atrial thrombus, as well as a positive correlation with haemodynamic abnormalities, suggesting that the hypercoagulable state may be related to atrial stasis and thrombus formation. Certainly, left atrial appendage thrombus may be present in up to $15 \%$ of patients with acute $\mathrm{AF}^{28}$ and the present study showing prothrombotic indices in acute AF is consistent with these observations.

To our surprise, we found no significant changes in STM, vWf, and fibrin D-dimer concentrations at days 1, 3, 7, and 30 after successful cardioversion of acute AF. This is in keeping with a recent report by Li Saw Hee and colleagues $^{17}$ that cardioversion of persistent AF did not significantly alter indices of hypercoagulability, even after three months' maintenance of sinus rhythm, despite the return of atrial systole. Importantly, Li Saw Hee and colleagues ${ }^{17}$ also reported that there were significant differences in the prothrombotic state when patients with paroxysmal and permanent AF were compared with matched patients with persistent AF or controls in sinus rhythm. Differences in study populations may account for the variable results noted in relation to cardioversion of AF.

Table 2 Plasma soluble thrombomodulin, von Willebrand factor, and fibrin D-dimer in patients with acute $\mathrm{AF}^{*}$, chronic $\mathrm{AF}$, ischaemic heart disease patients, and healthy controls

\begin{tabular}{llllll}
\hline & Acute AF* & Chronic AF & IHD & Healthy controls & p Value \\
\hline Number & 24 & 24 & 24 & 24 & \\
STM $(\mathrm{ng} / \mathrm{ml})$ & $12.1(4.1)$ & $11.8(4.6)$ & $7.4(3.7)$ & $5.9(2.7)$ & $<0.001$ \\
vWf $(\mathrm{ng} / \mathrm{ml})$ & $137.2(36.9)$ & $133.1(25.0)$ & $110.0(29.0)$ & $86.7(33.2)$ & $<0.001$ \\
DD $(\mu \mathrm{g} / \mathrm{ml})$ & $2.35(2.68)$ & $1.12(0.65)$ & $0.99(0.73)$ & $0.39(0.28)$ & $<0.001$ \\
\hline
\end{tabular}

*Samples from patients with acute AF were taken 30 days after sinus rhythm restoration. $D D$, fibrin D-dimer; sTM, soluble thrombomodulin; $v W F$, von Willebrand factor. Post hoc analyses: acute AF $v$ chronic AF: $\operatorname{sTM}(p=0.808), v W f(p=0.888), D D(p=0.038)$; acute AF vIHD: sTM $(\mathbf{p}<0.001), v W f(p=0.029), D D(p=0.030) ;$ acute AF $v$ controls: $\operatorname{sTM}(\mathbf{p}<0.001), v W f(p<0.001), D D(p<0.01)$ chronic AF $v$ IHD: sTM ( $<<0.01)$, vWf $(p<0.01), D D(p=0.574)$; chronic AF $v$ controls: sTM ( $p<0.001)$, vWf $(\mathbf{p}<0.001), \mathrm{DD}(\mathbf{p}<0.001) ; \mathrm{HD} v$ controls: $\operatorname{sTM}(p=0.149), \mathrm{vWf}(\mathbf{p}=0.017), \mathrm{DD}(\mathbf{p}<0.01)$ 
Table 3 Changes in Doppler echocardiographic and prothrombotic indices during follow up

\begin{tabular}{|c|c|c|c|c|c|}
\hline & Day 1 & Day 3 & Day 7 & Day 30 & p Value \\
\hline Peak E wave velocity $(\mathrm{m} / \mathrm{s})$ & $0.68(0.13)$ & $0.71(0.18)$ & $0.74(0.16)$ & $0.672(0.19)$ & 0.539 \\
\hline Peak A wave velocity $(\mathrm{m} / \mathrm{s})$ & $0.79(0.23)$ & $0.84(0.23)$ & $0.82(0.21)$ & $0.81(0.22)$ & 0.369 \\
\hline$E: A$ & $0.90(0.28)$ & $0.88(0.22)$ & $0.98(0.32)$ & $0.91(0.31)$ & 0.266 \\
\hline $\mathrm{A} / \mathrm{T}$ & $40.8(11.4)$ & $38.6(10.9)$ & $35.9(10.6)$ & $39.2(10.6)$ & 0.114 \\
\hline sTM (ng/ml) & $11.5(3.0)$ & $12.3(4.7)$ & $11.5(4.4)$ & $12.1(4.1)$ & 0.708 \\
\hline vWf (ng/ml) & $139.3(40.9)$ & $136.9(39.0)$ & $131.4(37.0)$ & $137.2(36.9)$ & 0.665 \\
\hline $\mathrm{DD}(\mu \mathrm{g} / \mathrm{ml})$ & $3.89(3.91)$ & $4.11(4.18)$ & $3.11(3.16)$ & $2.35(2.68)$ & 0.066 \\
\hline
\end{tabular}

Nonetheless, Sohara and colleagues ${ }^{8}$ reported that acute AF does not affect the coagulation system within an average of six hours after onset, but increased platelet activity and a prothrombotic state were present 12 hours after the beginning of the episode. Recently, Giansante and colleagues ${ }^{29}$ found increased concentrations of fibrinopeptide $\mathrm{A}$, a thrombin generation marker, in patients with paroxysmal $\mathrm{AF}$, which remained stable at 28 days after reversion to sinus rhythm, and concentrations were higher than among the controls. In contrast, Oltrona and associates ${ }^{30}$ described an early significant increase in prothrombotic markers 24 and 72 hours after pharmacological cardioversion of persistent AF. They concluded that cardioversion itself may cause a prothrombotic state. Additionally, Lip and colleagues ${ }^{16}$ found a significant reduction in fibrin D-dimer concentrations at day 14 after cardioversion of persistent AF, but this study was based on only seven patients.

This study is limited by the fact that many of the AF patients had underlying vascular disease. We do accept that the abnormal sTM, vWf, and fibrin D-dimer concentrations may reflect more generalised vascular disease, heart disease, or hypertension rather than AF per se, although previous works have shown that the prothrombotic state in AF is independent of underlying structural heart disease or aetiology (as reviewed by $\operatorname{Lip}^{4}$ ). The coexistence of underlying disease may in part account for the abnormal concentrations of these indices in our patients after acute AF, although the acute AF group still had much higher concentrations of sTM, vWf, and fibrin D-dimer than did the vascular disease controls, who were patients with previous myocardial infarction. Although we took great care to include only patients with a known onset of AF, the possibility remains that some of our patients had frequent and asymptomatic episodes of AF resulting in intermittent flow abnormalities and coagulation activation. Despite inconsistent antiplatelet treatment in the studied groups, previous data do not suggest a significant effect of aspirin on the measured indices. ${ }^{31} 32$ Lastly, we did not perform transoesophageal echocardiography in our patients with acute AF before cardioversion to specifically look for spontaneous echo contrast or left atrial appendage thrombus, as the main objective of our study was to correlate the improvement of left atrial function with the changes in prothrombotic markers after reversion to sinus rhythm.

In conclusion, patients after acute onset $\mathrm{AF}$ have an abnormal prothrombotic state, with endothelial damage or dysfunction (high sTM, vWf) and increased thrombogenesis (fibrin D-dimer), which do not appear to be significantly altered in the 30 days after cardioversion to sinus rhythm.

\section{ACKNOWLEDGEMENTS}

We thank Dr José Sánchez for his invaluable assistance with statistics.

\section{Authors' affiliations}

F Marín, V E Climent, A Ibáñez, A García, F Sogorb, Department of Cardiology, General Hospital of Alicante, Alicante, Spain
V Roldán, Haematology Unit, Hospital of San Vicente, Alicante, Spain P Marco, Department of Haematology, General Hospital of Alicante, Alicante, Spain

G Y H Lip, Haemostasis, Thrombosis and Vascular Biology Unit, University Department of Medicine, City Hospital, Birmingham, UK

\section{REFERENCES}

1 Atrial Fibrillation Investigators. Risk factors for stroke and efficacy of antithrombotic therapy in atrial fibrillation: analysis of pooled data from five randomized trials. Atrial fibrillation investigators. Arch Intern Med 1994; 154:1449-57

2 Laupacis A, Albers $G$, Dalen J, et al. Antithrombotic therapy in atrial fibrillation. Chest 1998;114:579S-89S.

3 Prystowsky EN, Benson W, Fuster V, et al. Management of patients with atrial fibrillation: a statement for healthcare professionals from the subcommittee on electrocardiography and electrophysiology, American Heart Association. Circulation 1996;93:1262-77.

4 Lip GYH. Does atrial fibrillation confer a hypercoagulable state? Lancet 1994;346:1313-4.

5 Roldán V, Marín F, Marco P, et al. Hypofibrinolysis in atrial fibrillation. Am Heart $J$ 1998; 136:956-60.

6 Kumagai K, Fukunami M, Ohmori M, et al. Increased intracardiovascular clotting in patients with chronic atrial fibrillation. J Am Coll Cardiol 1990; 16:377-80.

7 Marín F, Roldán V, Monmeneu JV, et al. Prothrombotic state and elevated levels of plasminogen activator inhibitor-1 in mitral stenosis with and without atrial fibrillation. Am J Cardiol 1999:84:862-4.

8 Sohara H, Amitani S, Kurose M, et al. Atrial fibrillation activates platelets and coagulation in a time-dependent manner: a study in patients with paroxysmal atrial fibrillation. J Am Coll Cardiol 1997;29:106-12.

9 Lip GYH, Lowe GDO, Rumley A, et al. Fibrinogen and fibrin D-dimer levels in paroxysmal atrial fibrillation: evidence for intermediate elevated levels of intravascular thrombogenesis. Am Heart J 1996;131:724-30.

10 Mattioli AV, Castelli A, Bastia E, et al. Atrial ejection force in patients with atrial fibrillation: comparison between DC shock and pharmacological cardioversion. Pacing Clin Electrophysiol 1999;22:33-8.

11 Manning WJ, Silverman DI, Katz SE, et al. Temporal dependence of the return of atrial mechanical function on the mode of cardioversion of atrial fibrillation to sinus rhythm. Am J Cardiol 1995;75:624-6.

12 Sparks PB, Jayaprakash S, Mond HG, et al. Left atrial mechanical function after brief duration atrial fibrillation. J Am Coll Cardiol 1999;33:342-9.

13 Fatkin D, Kuchar DL, Thorburn CW, et al. Transesophageal echocardiography before and during direct current cardioversion of atrial fibrillation: evidence for "atrial stunning" as a mechanism of thromboembolic complications. J Am Coll Cardiol 1994;23:307-16.

14 Lip GYH, Lowe GDO. Fibrin D-dimer: a useful clinical marker of thrombogenesis? Clin Sci 1995;89:205-14.

15 Fuster V, Rydén LE, Asinger RW, et al. ACC/AHA/ESC guidelines for the management of patients with atrial fibrillation; a report of the American College of Cardiology/American Heart Association task force on practice guidelines and the European Society of Cardiology committee for practice guidelines and policy conferences (committee to develop guidelines for the management of patients with atrial fibrillation) developed in collaboration with North American Society of Pacing and Electrophysiology. Eur Heart J 2001;22:1852-923.

16 Lip GYH, Rumley A, Dunn FG, et al. Plasma fibrinogen and fibrin D dimer in patients with atrial fibrillation: effects of cardioversion to sinus rhythm. Int J Cardiol 1995;51:245-51.

17 Li Saw Hee FL, Blann AD, Gurney AD, et al. Plasma von Willebrand factor, fibrinogen and soluble P-selectin levels in paroxysmal, persistent and permanent atrial fibrillation: effects of cardioversion and return of left atrial function. Eur Heart J 2001;22:1741-7.

18 Manning WJ, Leeman DE, Gotch BS, et al. Pulsed Doppler evaluation of atrial mechanical function after electrical cardioversion of atrial fibrillation. J Am Coll Cardiol 1989;13:617-23.

19 Grimm RA, Stewart WJ, Arheart KL, et al. Left atrial appendage "stunning", after electrical cardioversion in atrial flutter: an attenuated response compared with atrial fibrillation as the mechanism for lower susceptibility to thromboembolic events. J Am Coll Cardiol 1997;29:582-9.

20 Black IW, Fatkin D, Sagar KB, et al. Exclusion of atrial thrombus by transesophageal echocardiography does not preclude embolism after cardioversion of atrial fibrillation. Circulation 1994;89:2509-13. 
21 Omran $\mathrm{H}$, Jung $\mathrm{W}$, Rabahieh $\mathrm{R}$, et al. Left atrial chamber and appendage function after internal atrial defibrillation: a prospective and serial transesophageal echocardiographic study. $J$ Am Coll Cardiol 1997;29:131-8.

22 Falcone RA, Morady F, Armstrong WF. Transesophageal echocardiographic evaluation of left atrial appendage function and spontaneous contrast formation after chemical or electrical cardioversion of atrial fibrillation. Am J Cardiol 1996;78:435-9.

23 Grimm RA, Leung DY, Black IW, et al. Left atrial appendage "stunning" after spontaneous conversion of atrial fibrillation demonstrated by transesophageal Doppler echocardiography. Am Heart J 1995;130:174-6.

24 Kamp O, Verhorst PMJ, Visser CA. Transesophageal echocardiography for early cardioversion of atrial fibrillation. Am J Cardiol 2000:86:46G-50G.

25 Shirani J, Alaedini J. Structural remodeling of the left atrial appendage in patients with chronic atrial fibrillation: implications for thrombus formation, systemic embolism, and assessment by transesophageal echocardiography. Cardiovasc Pathol 2000;9:95-101.

26 Lip GYH, Lowe GOD, Rumley A, et al. Increased markers of thrombogenesis in chronic atrial fibrillation: effects of warfarin treatment. Br Heart $J$ 1995;73:527-33
27 Heppel RM, Berkin KE, McLechanan JM et al. Haemostatic and haemodynamic abnormalities associated with left atrial thrombosis in nonrheumatic atrial fibrillation. Heart 1997;77:407-1 1

28 Stoddard MF, Dawkins PR, Prince CR, et al. Left atrial appendage thrombus is not uncommon in patients with acute atrial fibrillation and a recent embolic event: a transesophageal echocardiographic study. J Am Coll Cardiol 1995;25:452-9.

29 Giansante C, Fiotti N, Miccio M, et al. Coagulation indicators in patients with paroxysmal atrial fibrillation: effects of electric and pharmacologic cardioversion. Am Heart J 2000; 140:423-9.

30 Oltrona L, Broccolino M, Merlini PA, et al. Activation of the hemostatic mechanism after pharmacological cardioversion of acute nonvalvular atrial fibrillation. Circulation 1997;95:2003-6.

31 Lip GYH, Lip PL, Zafiris J, et al. Fibrin D-Dimer and $\beta$-thromboglobulin as markers of thrombogenesis and platelet activation in atrial fibrillation: effects of introducing ultra-low-dose warfarin and aspirin. Circulation 1996;4:425-31

32 Kamath S, Blann AD, Chin BSP, et al. A prospective randomized trial of aspirin-clopidogrel combination therapy and dose-adjusted warfarin on indices of thrombogenesis and platelet activation in atrial fibrillation. J Am Coll Cardiol 2002;40:484-90.

\section{IMAGES IN CARDIOLOGY}

The utility of a comprehensive cardiac magnetic resonance examination for the evaluation of patients with heart failure

W report two cases of patients referred for assessment to a heart failure clinic. The first case is a 62 year old woman with a history of previous myocardial infarction. The second case is a 65 year old woman with a history of heart failure, chest pains, smoking, and hypertension. Both patients presented with symptoms of moderate-to-severe heart failure. Echocardiography in both cases was limited by suboptimal image quality but showed significant left ventricular (LV) dilatation with severe global and regional (septal dyskinesia) systolic dysfunction.

Investigation by cardiac magnetic resonance (CMR) imaging confirmed the presence of LV dilatation (LV end diastolic volume: case $1=230 \mathrm{ml}$, case $2=276 \mathrm{ml}$ ) and severe systolic dysfunction (LV ejection fraction: case $1=27 \%$, case $2=30 \%$ ). Unlike echocardiography, CMR provided high quality images demonstrating thinning of dysfunctional segments (sign of previous infarct) in case 1 and preserved myocardial thickness in case 2. CMR performed 10 minutes after an injection of gadolinium based contrast agent revealed areas of delayed enhancement corresponding to areas of myocardial scar. Extensive delayed enhancement was found in case 1 suggesting transmural postinfarction scar and no enhancement in case 2 indicating viable myocardium.

These cases demonstrate that a comprehensive CMR examination can provide accurate information on LV morphology and function in cases with suboptimal echocardiographic images and give important additional information on the extent of scar and myocardial viability in patients with LV systolic dysfunction. This may help identify patients for appropriate interventions.

N P Nikitin

$R$ de Silva J G F Cleland n.p.nikitin@hull.ac.uk
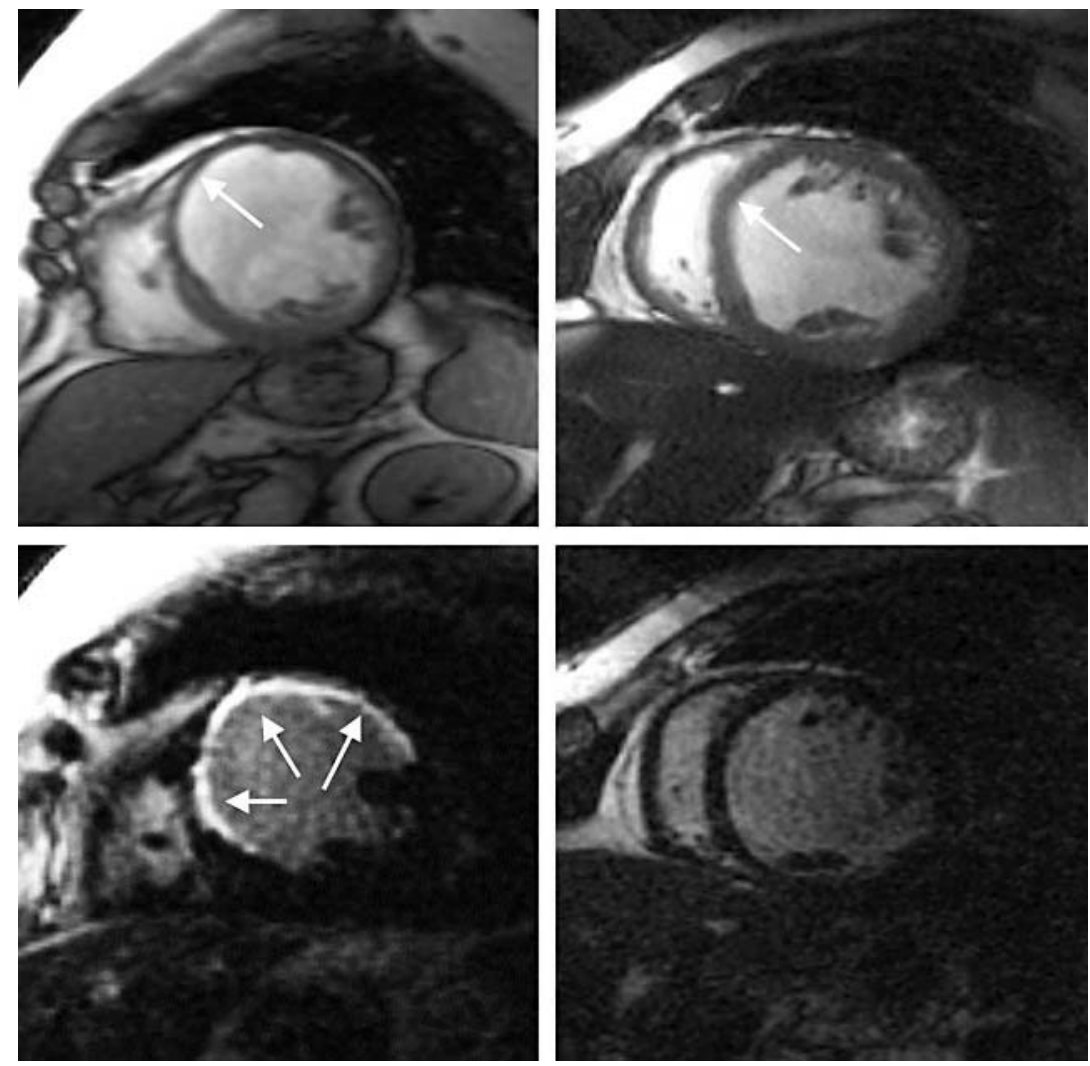

All images represent mid ventricular short axis views. Upper panels show end diastolic phases obtained using ECG triggered gradient echo cine CMR. Lower panels demonstrate contrast enhanced images obtained using a segmented inversion recovery fast gradient echo sequence. Upper left panel. Case 1: LV dilatation with myocardial thinning and dyskinesia (arrow) in anterior and anteroseptal segments. Upper right panel. Case 2: LV dilatation with wall paradoxical septal motion (arrow). Note preserved myocardial thickness of intraventricular septum. Lower left panel. Case 1: A contrast enhanced scan showing extensive transmural scarring (bright areas of contrast enhancement) in anteroseptal, anterior, and lateral segments (arrows). Lower right panel. Case 2 A contrast enhanced scan with no delayed enhancement indicating absence of myocardial scar. 